\title{
4D Flat Panel Conebeam CTA for In Vivo Imaging of the Microvasculature of the Human Cortex with a Novel Software Prototype
}

\author{
(D) N. Huizinga, (D) F. Keil, (D) A. Birkhold, (D). Kowarschik, (DS. Tritt, and (D). Berkefeld
}

\begin{abstract}
SUMMARY: It was the aim of our pilot study to investigate whether time-resolved flat panel conebeam CTA is able to demonstrate small cortical vessels in vivo. In 8 patients with small AVMs, time-resolved coronal MPRs of the vasculature of the frontal cortex were recalculated from 3D rotational angiography datasets with the use of a novel software prototype. 4D flat panel conebeam CTA demonstrated the course of the cortical arteries with small perpendicular side branches to the underlying cortex. Pial arterial and venous networks could also be identified, corresponding to findings in injection specimens. Reasonable image quality was achieved in 6 of 8 cases. In this small study, in vivo display of the cortical microvasculature with 4D flat panel conebeam CTA was feasible and superior to other angiographic imaging modalities.
\end{abstract}

ABBREVIATIONS: 3DRA $=3$ D rotational angiography; FPCBCTA $=$ flat panel conebeam CTA

$I_{\mathrm{d} e}^{\mathrm{n}}$ the past, the vascular supply of the human cortex has been described according to findings from ex vivo injection specimens. The cortical arteries follow the course of the sulci as a source of a pial network of anastomoses and small direct branches to the cortex. Cortical draining veins are also collected by a venous network with further connections into superficially located cortical veins. ${ }^{1}$ Beyond the level of pre- and postcapillary vessels, the vascular ultrastructure was investigated further by means of scanning electron microscopy, which was able to detect capillary networks within the cortical layers. ${ }^{2}$ In vivo vascular imaging with DSA is widely limited to the display of larger branches. Small arterial and venous networks of the cortex are frequently obscured by the overlay of vessels on projection images. The resolution of other, less invasive angiographic modalities such as CTA or MRA is even worse, and reliable display of submillimeter structures cannot be achieved with routine acquisitions. SWI has the potential to show the microvasculature at $7 \mathrm{~T}$ or with the use of iron-based contrast agents. ${ }^{3}$ Until now, the clinical interest in imaging of the microvasculature of the human cortex was limited, but this may change due to research demonstrating the importance of the microcirculation in patients with cerebral ischemia or smallvessel disease. $^{4-7}$

Received December 30, 2019; accepted after revision March 28, 2020. From the Institute of Neuroradiology (N.H., F.K., J.B.), University Hospital of Frankfurt, Frankfurt, Germany; Siemens Healthcare GmbH (A.B., M.K.), Forchheim, Germany; and Helios Dr. Horst Schmidt Kliniken Wiesbaden (S.T.), Wiesbaden, Germany.

The concepts and information presented in this article are based on research and are not commercially available.

Please address correspondence to Joachim Berkefeld, MD, Institute of Neuroradiology, University Hospital of Frankfurt, Schleusenweg 2-16, D-60528 Frankfurt am Main, Germany; e-mail: Berkefeld@em.uni-frankfurt.de

http://dx.doi.org/10.3174/ajnr.A6574
It was shown that flat panel conebeam CTA (FPCBCTA) derived from $3 \mathrm{D}$ rotational angiography (3DRA) datasets is able to demonstrate small perforating branches of proximal parts of the cerebral arteries. ${ }^{8,9}$

Recent technical advances have led to a more refined technique, with the possibility of using the temporal information of 3DRA to reconstruct time-resolved 3D volumes and CT-like images. ${ }^{10}$ It was the purpose of our pilot study to determine whether in vivo imaging of the cortical microvasculature is feasible with the use of $4 \mathrm{D}$-FPCBCTA. The $4 \mathrm{D}$ option was chosen due to the advantage to select and demonstrate the proper angiographic phase with the best filling of the vessels. The use of a novel software prototype facilitated the visualization of the consecutive filling of small arteries and veins.

\section{MATERIALS AND METHODS}

Time-resolved coronal MPRs of the vasculature of the frontal cortex were recalculated from routinely acquired 3DRA datasets. The patients had justifying indications for a 3DRA protocol with a prolonged scan time of 12 seconds for treatment planning of AVMs.

For evaluation of the normal microvasculature, we selected 8 consecutive patients with small supratentorial AVMs with a nidus size of $<3 \mathrm{~cm}$ and without major compromise of hemodynamics in the frontal cortex. Proceedings were approved by the local ethics committee at the University Hospital of Frankfurt, Germany.

All patients had selective transfemoral catheterization of the ICA under local anesthesia. DSA projection images and 3DRA datasets were obtained using modified Artis zee biplane neuroangiography equipment (Siemens). For 3DRA, the rotation angle was $260^{\circ}$ with 2 runs (a native run without contrast and a fill run with contrast, 304 projections each) of 12 seconds each. The acquisition protocol is based on $2 \times 2$ binning of the 
detector pixels of $154 \times 154 \mu \mathrm{m}$ each $(0.308 \times 0.308 \mathrm{~mm}$ effective pixel size). The FOV of the reconstructions was $238 \times$ $184 \mathrm{~mm}$ with an isotropic voxel size of $0.46 \mathrm{~mm}^{3}$ and a $512^{2} \mathrm{ma}-$ trix. After an x-ray delay of 2 seconds in the fill run, $20 \mathrm{~mL}$ of nonionic contrast with $300 \mathrm{mg}$ of iodine $/ \mathrm{mL}$ was automatically injected with a flow rate of $3 \mathrm{~mL} / \mathrm{s}$. For 2D-DSA, we used a protocol with a variable frame rate with up to 2 frames/s after hand injection of contrast.

For the reconstruction of $4 \mathrm{D}$ volumes and corresponding MPRs, the projection images were transferred to a dedicated workstation (syngo X Workplace VD20; Siemens) equipped with a software prototype as an extension of the currently available syngo Dyna4D software (Siemens). The prototype reduces streak artifacts, and the reconstruction algorithm should improve the recognition of consecutive filling of arteries and veins. We evaluated the cortical microvasculature, according to 4 D-FPCBCTA MPRs in a coronal plane perpendicular to the course of the frontal gyri and sulci. The MPR with the best filling of small vessels was selected according to a cine display of the sequence of time-resolved MPRs, which is available at any time point during the scan time of 12 seconds. Usually the best filling of the microvasculature was visible after two-thirds of the scan time and at the end of the scan. We also tried MIPs or cuts from volume rendering data, which were found to be inferior to MPRs. The best visualization of the tubular character of small vessels occurred from section thicknesses between 6 and $9.5 \mathrm{~mm}$. The findings from the reconstructions were compared with corresponding DSA images in a posterioranterior projection and with findings in the literature.

Identification of vascular structures and image documentation was by consensus of 2 reviewers.

\section{RESULTS}

Eight consecutive patients ( 7 men and 1 woman, with a mean age of 45 years) met the inclusion criteria. Two datasets were only partly evaluable due to incomplete filling of the anterior cerebral artery due to hypoplasia of the A1 segment or movement artifacts.

In 6 fully evaluable cases, 4D-MPRs demonstrated the main branches of the cortical arteries following the course of the gyri into the depth of the sulci. From these main branches, small perpendicular arteries were visible, which could be followed for a few millimeters in the direction of the cortex. Intracortical networks were not detectable. Beneath these direct cortical branches, we found network-like anastomoses of small arteries above the surface of the cortex (Fig 1). In the venous phase, cortical draining veins could be identified perpendicular to larger collecting veins with network-like structures draining further into straight veins toward superficial cortical veins (Fig 2). The findings on MPRs were confirmed on corresponding DSA images with their sharper separation of the angiographic phases. Comparisons with drawings derived from illustrations from the literature ${ }^{1,2}$ also confirmed our main findings.

\section{DISCUSSION}

Our casuistic pilot study showed the feasibility of in vivo imaging of the cortical microvasculature with the use of 4D-FPCBCTA and a novel software prototype. Compared with conventional reconstructions from 3D volume data, time-resolved MPRs are advantageous because they enable selection of the proper volume in the angiographic phases with the best filling of small arteries or veins. As is known from previous studies, temporal resolution of 4D-FPCBCTA and derived reconstructions is limited by overlapping of the arterial and venous phases. ${ }^{11}$

The filling of smaller vessels with slower flow demands a prolonged contrast bolus covering larger parts of the scan time compared with the shorter and sharper bolus of 2D-DSA. Even with the use of the software prototype, the overlapping angiographic phases led to difficulties in differentiating arteries and veins. Therefore, we selected reconstructions with typical anatomic patterns described for the course of arteries following the gyri and sulci, pial networks, and veins. The veins could be identified due to enhanced filling at the end of the scan and due to their straight course and connections to cortical veins, which could easily be detected. Correct assignment of arteries and veins was confirmed by comparison with DSA images showing the main structures. The display of fine details, such as pial or venous networks, was disadvantageous on DSA projection images due to the overlay of adjacent cortical vessels and their territories. In the current prototype implementation, the same threshold is used for segmentation of arteries and veins. Future implementations may investigate a thresholding technique for distinguishing arteries and veins.

The spatial resolution of $4 \mathrm{D}$-FPCBCTA is limited; thus, we were unable to demonstrate intracortical vessels and vascular networks with vessels smaller than $100 \mu \mathrm{m}$, which have been previously described in scanning electron microscopy studies. ${ }^{2}$ Only stump-like proximal parts of intracortical arteries and veins could be visualized. The vessel sizes we could display ranged between 150 and $750 \mu \mathrm{m}$, according to descriptions in the literature. ${ }^{1,2}$ In animal experiments, MR imaging with SWI and iron-based contrast agents could visualize intracortical vessels of $>10 \mu \mathrm{m} .{ }^{3}$ For optimal display of vessels, section thicknesses between 6 and $9.5 \mathrm{~mm}$ were chosen. Thinner slices could improve the detection of small branches. The tubular character and branching of the vessels are better displayed on thick-section MPRs.

For our study, we used an Artis zee system (Siemens), which is not the latest technology. The visualization of vessels smaller than the voxel size of $0.5 \mathrm{~mm}^{3}$ may be challenging, even with the latest generations of neuroangiography systems. Secondary reconstructions with smaller voxel sizes may improve the visualization of small vessels.

Based on our experience with the software prototype, further improvement of image quality in the display of small vessels can be expected and may become the subject of future studies.

Suboptimal image quality was caused by incomplete filling of the anterior cerebral territory in a patient with a hypoplastic A1 segment. FPCBCTA after IV contrast could avoid this problem of selective injections. In tentative reconstructions, the opacification of small vessels after IV contrast was much weaker and hardly evaluable.

Movement artifacts caused image degradation in another patient. General anesthesia, which could further improve image quality, is not adequate for a purely diagnostic procedure for treatment planning of an AVM. We tried reconstructions of 

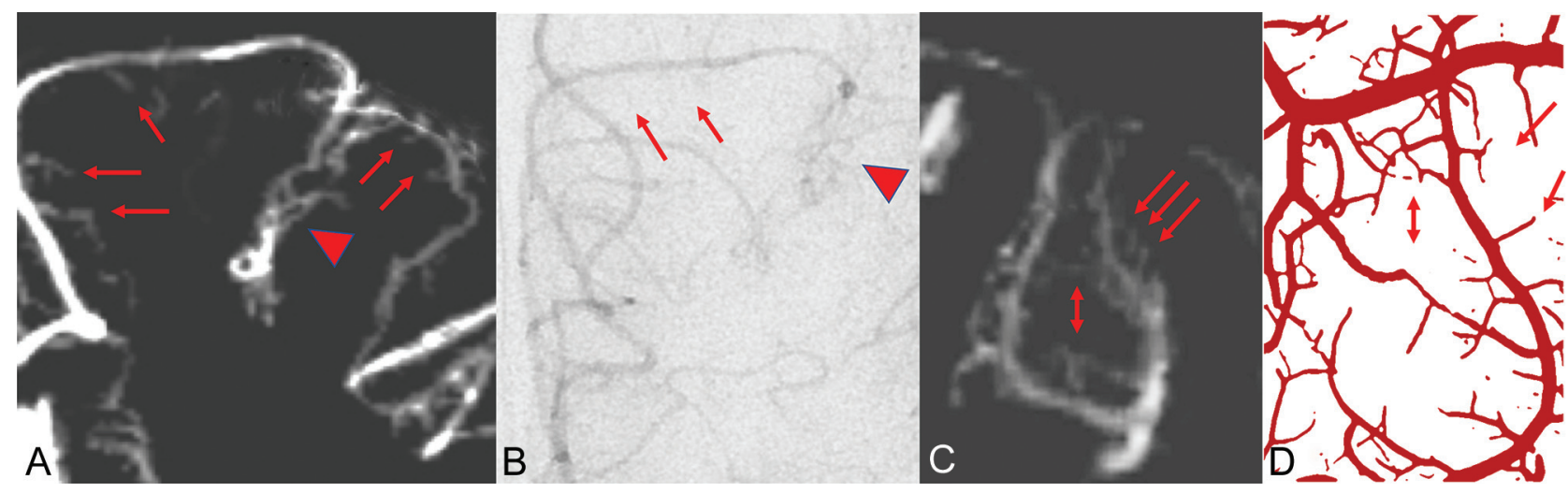

FIG 1. Coronal 4 D-FPCBCTA reconstruction of the arterial microvasculature at the superior and middle frontal gyri and the superior frontal sulcus $(A)$ and the corresponding DSA image, late arterial phase $(B)$. Main arterial branches (red arrows) and the sulcal pial arterial network (red arrowhead) can be identified. Perpendicular branches to the cortex are difficult to see on DSA. C, A more detailed view shows arteries bending from the cortical surface into the depth the superior frontal sulcus. Perpendicular branches can be followed in the direction of the cortex of the adjacent middle frontal gyrus (red arrows). Note network-like intra-arterial connections (double red arrow). $D$, Similar findings are detected in injection specimens of cortical arteries (corresponding arrows). Drawing reprinted with permission from Duvernoy et al.
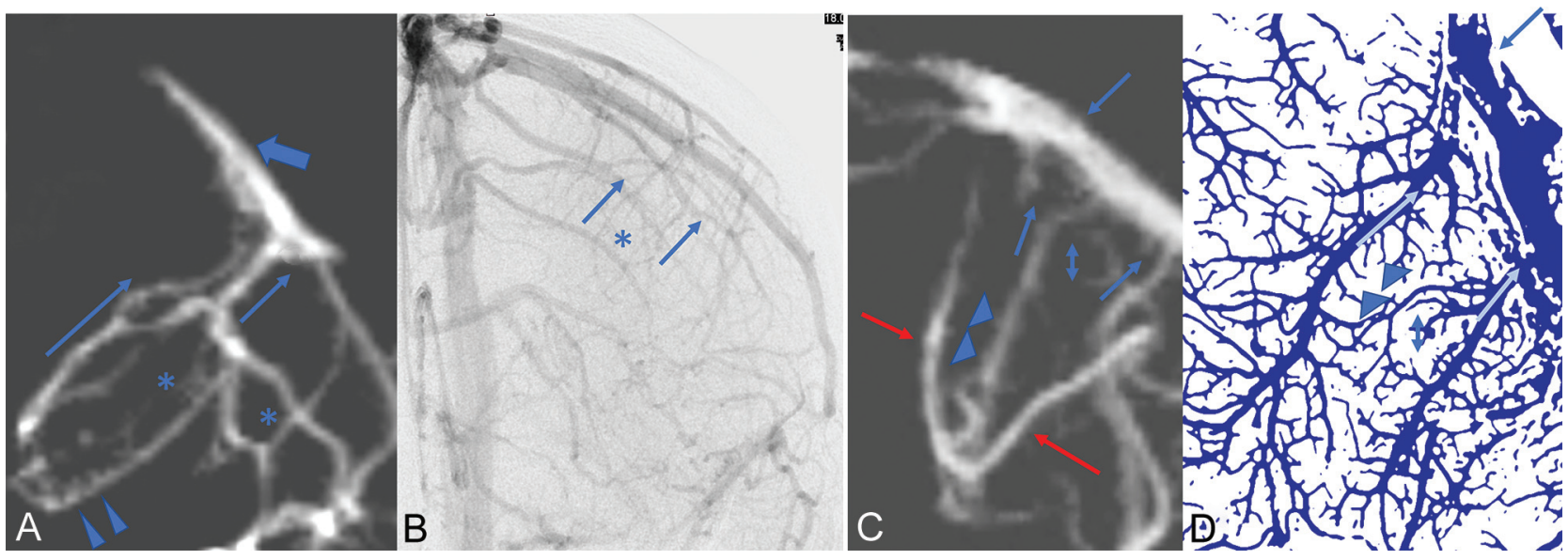

FIG 2. Coronal 4D-FPCBCTA reconstruction of veins in the middle frontal sulcus $(A)$ and corresponding DSA image, late venous phase $(B)$. Collecting veins (thin blue arrows) and the sulcal venous network (asterisks) can be identified on DSA. Small veins draining into the collecting veins are only partly visible (blue arrowheads). Further drainage of the collecting veins into a superficial cortical vein (thick blue arrow). Note considerable overlay of venous structures in the DSA projection image. C, Cortical veins are also fed by perpendicular branches from the cortex (blue arrowheads). Collecting veins show a straight course toward a larger cortical vein (blue arrows). Network-like connections are weakly accentuated (blue double arrow). Note the overlay by a sulcal artery (red arrows). D, Corresponding findings on a drawing from an injection specimen with small cortical draining veins (blue arrowheads) perpendicular to straight collecting veins to a cortical vein (thin blue arrows). Drawing reprinted with permission from Duvernoy et al.'

3DRA data from patients with aneurysms under general anesthesia with scan times of 5 seconds. Display of the microvasculature was clearly inferior, probably due to incomplete filling of small vessels and a lack of temporal resolution.

A disadvantage of $4 \mathrm{D}$-FPCBCTA is its invasiveness, with potential risks of arterial catheterization. Until now, selective angiography with $4 \mathrm{D}$-FPCBCTA provides the best contrast resolution for small vessels compared with less invasive imaging modalities. ${ }^{12,13}$ One reason is the ability to obtain secondary reconstructions from the raw data without any loss of spatial resolution.

SWI after administration of iron-based contrast material may be further developed as a less invasive alternative. The first published images of the cerebral microvasculature with the use of this technique are promising but still inferior regarding the details seen on our reconstructions. ${ }^{3}$

Another disadvantage of $4 \mathrm{D}$-FPCBCTA is the increased radiation exposure of 3DRA, with a prolonged scan time of 12 seconds. Effective dosage, according to the International Commission on Radiological Protection 103 (ATOM Phantom; CIRS; mobile MOSFET dosimetry system, Best Medical Canada), of $0.94 \mathrm{mSv}$ for the 12 -second $4 \mathrm{D}$-FPCBCTA acquisition is lower than the reported doses of time-resolved CTA with the use of multislice $\mathrm{CT}^{14}$ but higher than actual published doses of 3DRA with shorter scan times. ${ }^{15}$ For 2D-DSA with 2 frames/s (Plane A - PosteriorAnterior, Plane B $-90^{\circ}$ to plane A), an effective dose of $0.41 \mathrm{mSv}$ (for 20 seconds) was determined. 
Prolonged scan time and temporal resolution are key factors for successful display of small vessels. One main limitation of our study is the small number of cases due to time-consuming postprocessing with manual window settings to enhance the vessels at the limits of the spatial resolution. The evaluation of a larger and more representative sample demands a standardization of the generation of 4D-MPRs, including further software improvement for tracking arteries and veins, which is challenging due to the close proximity of the arterial and venous parts of the cortical microvasculature. A more detailed comparison of the data with anatomic specimens, including measurements of vessel sizes, is also desirable.

Potential clinical applications of imaging of small cortical vessels are improved anatomic visualization of leptomeningeal anastomoses in patients with obstructions of cerebral arteries or in the surroundings of vascular malformations. Imaging of the normal microvasculature may also enhance our understanding of the angioarchitecture of pial AVMs. ${ }^{11}$ Current imaging studies in patients with small-vessel disease are focused on MR imaging at high field strengths to show subcortical microinfarcts or microbleeds. ${ }^{4,16}$ Visualization of small vessels is still based on postmortem studies. ${ }^{17}$ It would be interesting to know whether direct visualization of cortical vessels in vivo could contribute to assessment of vascular density or other pathologic changes in patients with degenerative or inflammatory small-vessel disease.

\section{CONCLUSIONS}

In this small study, in vivo imaging of the cortical microvasculature with 4D-FPCBCTA and the use of a novel software prototype were feasible. Spatial resolution is currently superior to other angiographic imaging modalities.

Disclosures: Fee Keil-RELATED: Payment for Writing or Reviewing the Manuscript: funding by Siemens, €750/publication paid to the university*; OTHER RELATIONSHIPS: The Workstation used for analyzing the data belongs to Siemens and was only given as a loan to our institution; cooperation between Siemens and the Institution of Neuroradiology Frankfurt with external funding of $€ 750 /$ publication for open access. Annette Birkhold—UNRELATED: Employment: Siemens Healthcare AG. Markus Kowarschik-UNRELATED: Employment: Siemens Healthineers AG, Comments: full-time employee. Stephanie Tritt-RELATED: Support for Travel to Meetings for the study or Other Purposes: There is a permanent scientific cooperation between Siemens and the Institute of Neuroradiology, Frankfurt, and travel fees to meetings were paid by the company.* Joachim Berkefeld—RELATED: Consulting Fee or Honorarium: scientific cooperation with Siemens Healthineers, Comments: 2.500, Eposterior-anterior paid to the university.* The dedicated workstation for $4 D$ reconstructions and the software prototype were provided by Siemens Healthineers*; UNRELATED: Consultancy: MicroVention; proctoring for endovascular treatment of intracranial aneurysms with the Woven Endo-Bridge (WEB)-device, Comments: 3000, _ posterior-anterior honorarium. *Money paid to the institution.

\section{REFERENCES}

1. Duvernoy HM, Delon S, Vannson L. Cortical blood vessels of the human brain. Brain Res Bull 1981;7:519-79 CrossRef Medline
2. Reina-De La Torre F, Rodriguez-Baeza A, Sahuquillo-Barris J. Morphological characteristics and distribution pattern of the arterial vessels in human cerebral cortex: a scanning electron microscope study. Anat Rec 1998;251:87-96 CrossRef Medline

3. Wang H, Jiang Q, Shen Y, et al. The capability of detecting small vessels beyond the conventional MRI sensitivity using iron-based contrast agent enhanced susceptibility weighted imaging. NMR Biomed 2020;33:e4256 CrossRef Medline

4. Wardlaw JM, Smith EE, Biessels GJ, et al; STandards for ReportIng Vascular changes on nEuroimaging (STRIVE v1). Neuroimaging standards for research into small vessel disease and its contribution to ageing and neurodegeneration. Lancet Neurol 2013;12:82238 CrossRef Medline

5. Kremer PH, Jolink WM, Kappelle LJ, et al; SMART and ESPRIT Study Groups. Risk factors for lobar and non-lobar intracerebral hemorrhage in patients with vascular disease. PLoS One 2015;10: e0142338 CrossRef Medline

6. Shi Y, Wardlaw JM. Update on cerebral small vessel disease: a dynamic whole-brain disease. Stroke Vasc Neurol 2016;1:83-92 CrossRef Medline

7. Shibuya M, da Costa Leite C, Lucato LT. Neuroimaging in cerebral small vessel disease: update and new concepts. Dement Neuropsychol 2017;11:336-42 CrossRef Medline

8. Lescher S, Samaan T, Berkefeld J. Evaluation of the pontine perforators of the basilar artery using digital subtraction angiography in high resolution and $3 \mathrm{D}$ rotation technique. AJNR Am J Neuroradiol 2014;35:1942-47 CrossRef Medline

9. Lescher S, Zimmermann M, Konczalla J, et al. Evaluation of the perforators of the anterior communicating artery (AComA) using routine cerebral $3 \mathrm{D}$ rotational angiography. J Neurointerv Surg 2016;8:1061-66 CrossRef Medline

10. Lang S, Gölitz P, Struffert T, et al. 4D DSA for dynamic visualization of cerebral vasculature: a single-center experience in 26 cases. AJNR Am J Neuroradiol 2017;38:1169-76 CrossRef Medline

11. Lescher S, Gehrisch S, Klein S, et al. Time-resolved 3D rotational angiography: display of detailed neurovascular anatomy in patients with intracranial vascular malformations. J Neurointerv Surg 2017;9:887-94 CrossRef Medline

12. Zhang Z, Fan Z, Kong Q, et al. Visualization of the lenticulostriate arteries at $3 \mathrm{~T}$ using black-blood $\mathrm{T} 1$-weighted intracranial vessel wall imaging: comparison with 7T TOF-MRA. Eur Radiol 2019;29:145259 CrossRef Medline

13. Nagata H, Murayama K, Suzuki S, et al. Initial clinical experience of a prototype ultra-high-resolution CT for assessment of small intracranial arteries. Jpn J Radiol 2019;37:283-91 CrossRef Medline

14. Radon MR, Chandran A, Bhojak M, et al. Radiation dose reduction in $4 \mathrm{D}$ cerebral CT angiography by individualized estimation of cerebral circulation time. AJNR Am J Neuroradiol 2016;37:2189-94 CrossRef Medline

15. Guberina N, Lechel U, Forsting M, et al. Dose comparison of classical 2-plane DSA and 3D rotational angiography for the assessment of intracranial aneurysms. Neuroradiology 2016;58:673-78 CrossRef Medline

16. Banerjee $G$, Wilson $D$, Jäger $H R$, et al. Novel imaging techniques in cerebral small vessel diseases and vascular cognitive impairment. Biochim Biophys Acta 2016;1862:926-38 CrossRef Medline

17. Kövari E, Herrmann FR, Gold G, et al. Association of cortical microinfarcts and cerebral small vessel pathology in the ageing brain. Neuropathol Appl Neurobiol 2017;43:505-13 CrossRef Medline 